\title{
B-Cell Linear Epitope Prediction on VP2 Partial Coat Protein Predicted Model of CPV-2b Isolate using Bioinformatics
}

\author{
Shalini Sharma ${ }^{1 *}$, Praseenjit Dhar ${ }^{1}$, Inderjeet Singh Yadav ${ }^{2}$ and Mandeep Sharma ${ }^{1}$ \\ ${ }^{1}$ Department of Veterinary Microbiology, College of Veterinary and Animal sciences, \\ CSKHPKV Palampur, Himachal Pradesh, India \\ ${ }^{2}$ School of Animal Biotechnology, PAU, Ludhiana, Punjab, India \\ *Corresponding author
}

\begin{abstract}
A B S T R A C T
Canine parvovirus is the major cause of haemorrhagic gastroenteritis in canines worldwide. There is a high morbidity and mortality in the young puppies. The currently available CPV vaccine is protecting from all antigenic strains but still there is vaccination failure as the strains are emerging at higher rate. The VP2 coat protein is the major coat protein it contains many B-cell epitopes against which many neutralizing antibodies can form, this structural protein of parvovirus can produce virus-like particles (VLPs) by a self-assembly process in vitro, making VLPs attractive vaccine candidates. So in this study a predicted protein model of partial protein coat of canine parvovirus isolate obtained after PCR detection and cell culture adaptation is made along with the display of B cell linear epitopes using bioinformatics tools. The protein sequence is deduced from the nucleotide sequence of the isolate obtained after sequencing. The length of nucleotide sequence of partial coat protein of CPV-2b isolate was 424 bp long the translated protein was 125 amino acid long and the B-Cell linear epitopes lies between the 59-75 amino acid positions. So in conclusion B-Cell epitope prediction on 3D model of CPV-2b isolate will be helpful in the vaccine design and for the production of therapaeutic antibodies moreover immunoinformatics is time saving does not requires extensive labour and animal experimentation.
\end{abstract}

Keywords

Epitopes,

CPV-2b and PDB

Article Info

Accepted:

10 March 2020

Available Online:

10 April 2020

\section{Introduction}

The CPV is a widespread virus that causes gastroenteritis and myocarditis in dogs. The infection is characterized by an acute phase, which appears shortly after the viruses invaded. As with other parvovirus, CPV replication is the most deleterious in actively dividing tissues (Parrish 1990). For puppies that do not have antibodies against the virus, CPV infections are devastating. Parvoviruses have a single-stranded DNA genome encapsidated in a $\mathrm{T} \quad 51 \quad\left(\begin{array}{lll}60 & \text { subunits })\end{array}\right.$ icosahedral capsid, $25 \mathrm{~nm}$ in diameter (Cotmore and Tattersal, 1987; Paradiso et al., 1982). Structurally and genetically related to feline panleukopenia virus, canine parvovirus (CPV) is a small non-enveloped, single 
stranded DNA virus. Capsids of CPV are assembled from three viral proteins (VP1, VP2, and VP3). In a viral infection, the massy capsid participates in the entire viral infection process. The capsid protein VP1 is primarily responsible for the infectivity of the virus, and the nuclear localization signal (NLS) of the VP1 serves as a guide to assist the viral genome in locating the nucleus.

The dominant protein VP2 provides an "antireceptor", which interacts with the cellular receptor and leads to the further internalization of virus, and, the N-terminal of VP2 also cooperates with the VP1 to prompt the process of nucleus translocation. VP2 is the major capsid protein which consists of an eight-stranded antiparallel $\beta$-barrel motif with four large insertions between $\beta$-strands called loops, containing many B-cell epitopes (Tsao et al., 1991). Epitope mapping experiments show that all epitopes generating neutralizing antibody are within VP2 (Turiso et al., 1991).

Several B-cell epitopes of amino terminus (Nterminus) of VP2 protein were defined by monoclonal antibodies (Langeveld et al., 1991). Additionally, a cleavage protein VP3 is a part of the capsid, which exists only in several members of the parvovirus family; however, the function of this cleavage protein remains to be fully determined. Resistance to the consequences of parvovirus infection is mediated by antibody specific for the parvovirus capsid proteins (Turiso et al., 1992).

For the feline parvoviruses, the loops between the B and C (loop 1), E and F (loop 2), and G and $\mathrm{H}$ (loops 3 and 4) $\beta$-strands contain 36, 74 , and 223 amino acids, respectively (Tsao et al., 1991). The four loops make up much of the surface of the virus capsid and comprised 364 of the 547 residues resolved in the VP2 structure, with loops 3 and 4 forming the bulk of the spikes that extend $22 \AA$ radially outwards around the threefold axes. The spikes contain many of the virus B-cell epitopes, being the immunodominant domains of the capsid (Strassheim et al., 1994).

Parvoviruses can suffer from the extreme environmental conditions such as low $\mathrm{pH}$, or even escape from the recognition of pattern recognition receptors (PRRs), due to the protection of the stable capsid, so the detection and determination of the capsid proteins is necessary, protein structure prediction is the inference of the threedimensional structure of a protein from its amino acid sequence - that is, the prediction of its folding and its secondary and tertiary structure from its primary structure.

Protein structure prediction is one of the most important goals pursued by bioinformatics and theoretical chemistry; it is highly important in canine medicine for example, in drug design and in the vaccine design. It also act as a target for the production of therapaeutic antibodies. Keeping this in view in this article Linear B cell epitope prediction on the predicted protein model of the partial coat protein VP2 of CPV-2b cell culture adapted isolate is performed.

\section{Materials and Methods}

In this study 102 faecal samples from dogs having haemorrhagic gastroenteritis were screened by CPV-2ab PCR assay targeting VP2 partial coat protein of canine parvovirus. Three positive faecal samples confirmed by PCR were processed for cell culture along with positive and negative controls. CPV isolate was prepared for sequencing, DNA from the cell culture adapted isolate was extracted by the phenol chloroform isoamyl alcohol method and PCR was performed. The PCR products were purified by using HiPurA ${ }^{\text {TM }}$ PCR Product Purification Spin Kit (Hi-media). The quantification of purified 
DNA was carried out in Nanodrop 1000 spectrophotometer and absorbance of DNA was recorded in UV range from 260/280 $\mathrm{nm}$. Values of Amax, Amin (A260/A280) were calculated to know the appropriate concentration. $45 \mu \mathrm{l}$ of DNA containing a total concentration of 1 to $2 \mu \mathrm{g}$ were sequenced of VP2 structural protein gene.

\section{Sequence analysis}

ExPASy translate (https://web.expasy.org/ translate/) was used to deduce the encoding protein from sequence of nucleotides. Three dimensional protein structure was modeled with homology based modelling program Modeller 9.23 (Fiser et al., 2003) based on the spatial restraints of protein structures using the PDB ID 1P5W as template. The quality of final model was assessed with Ramachandran plot (http://www.ebi.ac.uk/thornton-srv/databases/ pdbsum/Generate.html).

A comparison with already available 3D structure of VP2 model in PDB (1P5W:A PDBID) was done using Chimera extensive molecular modelling system (Pettersen EF, et al., 2004) . B cell linear epitope prediction of CPV-2b isolate was done using LBtop linear $\mathrm{B}$ cell epitope prediction server on the basis of SVM Score (Singh H et al., 2013).

\section{Results and Discussion}

Among the total 102 faecal samples targeted for VP2 structural protein only 52 showed positive PCR assay. Among the different antigenic types of CPV only CPV-2b was prevalent in this study and the isolation of CPV-2b was done successfully on MDCK for one faecal sample.

Sequencing of the PCR product resulted in 424 bp nucleotide sequence. The amino acid sequence was deduced from the nucleotide sequence using ExPASy translate. The translated protein was 125 amino acid long. Structure of homologous protein (PDB id: 1P5W) was used to build the structure of deduced protein from the nucleotide sequence.

Five protein models were generated. The model with lowest modeller DOPE score was selected and its quality was accessed using the Ramachandran plot in Figure 1. Plot showed that $99 \%$ residues were in allowed region and only one residue was in disallowed region.

Then a comparison of VP2 3D model with the template 1P5W showed a RMSD of $0.79 \AA$, demonstrating the higher similarity of the structures Figure 2.

LB top predicts the linear B cell epitope using the probalistic SVM model. LBtop identified a single B-Cell epitope "LKPRLHVNA PFVCQNNC" with higher probability. The epitope sequence was selected and displaced on the predicted protein model of partial coat protein of $\mathrm{CPV}-2 \mathrm{~b}$ isolate in the chimera demonstrate its presence on the protein surface and can be used for design of future vaccines. The translated protein was 125 amino acid long and B-cell epitopes lies in between the 59-75 amino acid positions Figure 3.

Epitopes are of particular interest to both clinical and basic biomedical researchers as they hold huge potential for vaccine design, disease prevention, diagnosis, and treatment.

It complements laboratory bench experimentation and has the potential to enhance greatly research productivity by unravelling complexity, saving time and reducing financial considerations (Masoud et al., 2015). B cell epitopes are recognized by B cell receptors or antibodies in their native structure. 


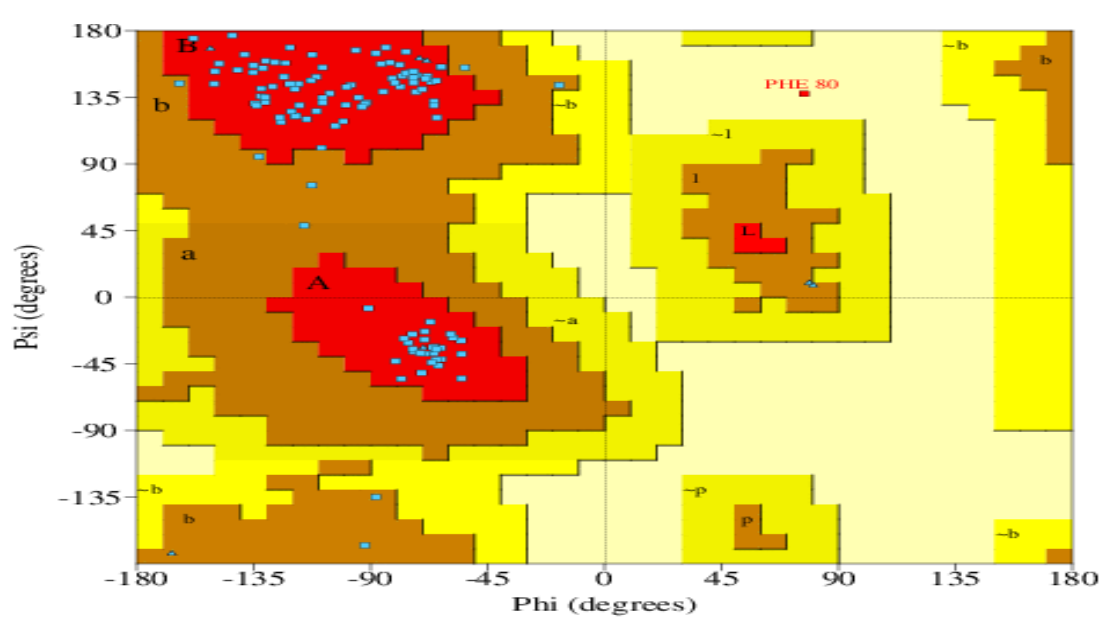

Fig.1 Ramachandran plot for protein model quality assessment

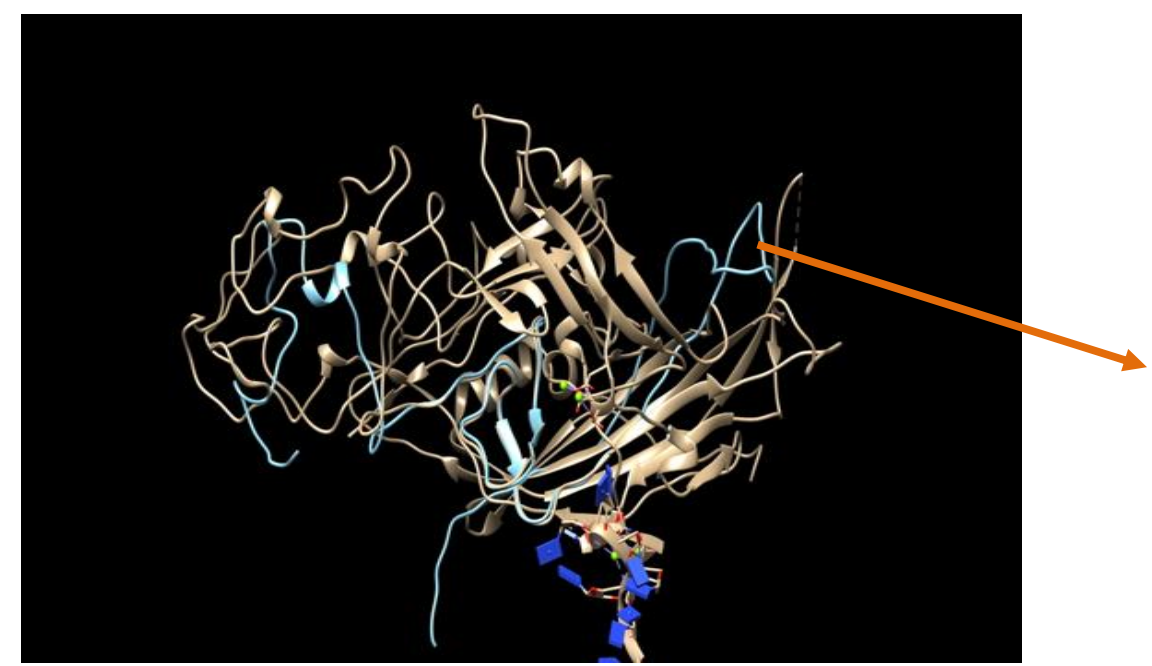

CPV-2b isolate PDB id:e714

Fig.2 Homologous Protein Image (1P5W:A PDB i.d sequence and CPV-2b isolate PDB id:e714) in Chimera Extensive Molecular Modelling System

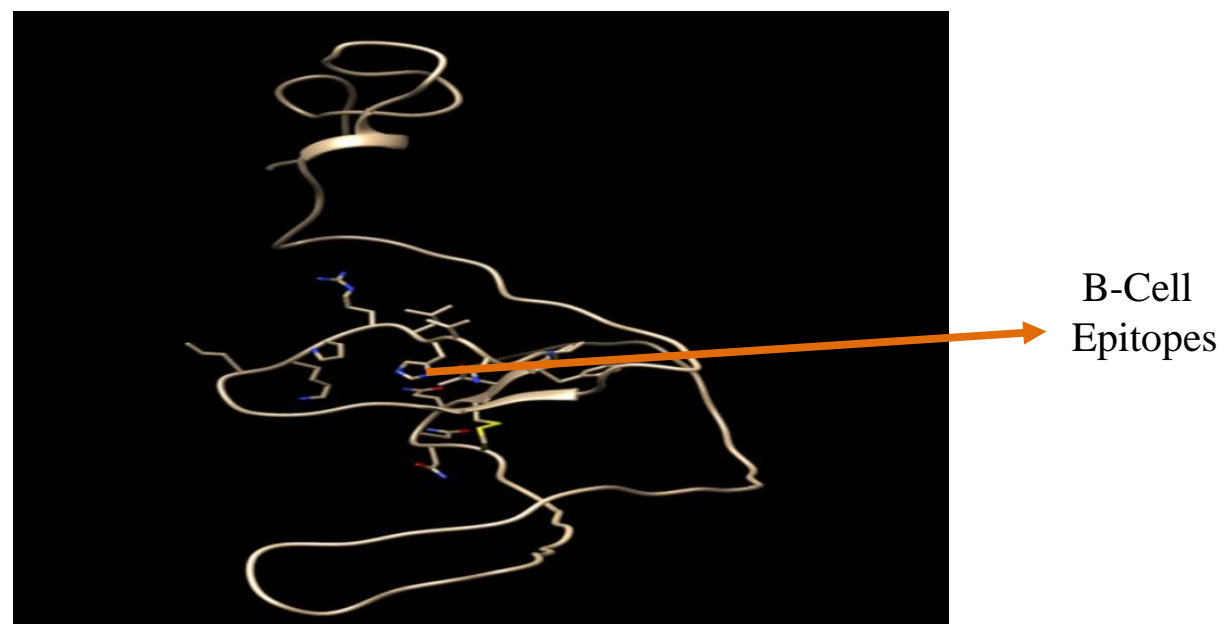

Fig.3 B-cell linear epitopes are in between (59-75) amino acid positions on the predicted protein model of partial coat protein VP2 of CPV-2b isolate (125) amino acid long 
VP2 Coat protein of canine parvovirus is the major and most abundant structural protein, accounting for $90 \%$ of the viral capsid, and is able to self-assemble to make virus like particles (VLPs) (Feng et al., 2014) it contains many B-cell epitopes that generates neutralizing antibody, Several B-cell epitopes of amino terminus (N-terminus) of VP2 protein were defined by monoclonal antibodies. Keeping these studies in view partial coat protein VP2 of CPV-2b isolate is targeted and studied. A similar study of Homology modeling and Molecular images of the major capsid protein of parvoviruses generated in UCSF Chimera (version 1.10.1) is done by (Callaway et al., 2016).

B-Cell linear epitope prediction on VP2 partial coat protein using predicted model of CPV-2b isolate with bioinformatics tool will be helpful for the vaccine design and for the generation of the therapeutic antibodies.

\section{Acknowledgements}

Author is thankful to CSKHPKV for providing necessary funds for the experiment. Author is highly thankful to the School of Bioinformatics PAU Ludhiana for necessary guidance.

\section{References}

Callaway HM, Feng KH, Lee DW, Allison AB, Pinard M, McKenna R, Agbandje McKenna M, Hafenstein S, Parrish CR. 2017. Parvovirus capsid structures required for infection: mutations controlling receptor recognition and protease cleavages. J Virol 91:e0187116. https://doi.org/10.1128/ JVI.0187116.

Cotmore, S. F., and Tattersal, P. (1987). The autonomously replicating parvoviruses of vertebrates. Adv. Virus Res. 33, 91174.
Feng H, Hu G-q, Wang H-l, Liang M, Liang $\mathrm{H}$, Guo H, et al., (2014) Canine Parvovirus VP2 Protein Expressed in Silkworm Pupae Self-Assembles into Virus-Like Particles with High Immunogenicity. PLoS ONE 9(1): e79575.https://doi.org/10.1371/journal.p one.0079575

Fiser A, Sali A (2003). Modeller: generation and refinement of homology-based protein structure models. Meth. Enzymol. Methods in Enzymology. 374. pp. 461-91.

Langeveld, J., Casal, J. I., Vela, C., Dalsgaard, K., Smale, S. H., Puijk, W. C., and Meloen, R. H. (1993). B cell epitopes of canine parvovirus: Distribution on the primary structure and exposure on the viral surface. J. Virol. 67, 765-772.

Lopez de Turiso JA, Cortes E, Martinez C et al., Recombinant vaccine for canine parvovirus in dogs. J Virol 1992; 66:2748-53.

Lopez de Turiso, J. A., Cortes, E., Ranz, A., Garcia, J., Sanz, A., Vela, C. and Casal, J. I. (1991). Fine mapping of canine parvovirus B cell epitopes. Journal of General Virology 72, 244-2456.

Masoud M, Taylor-Robinson AW (2015) Prediction of Epitopes of Viral Antigens Recognized by Cytotoxic $\mathrm{T}$ Lymphocytes as an Immunoinformatics Approach to Anti-HIV/AIDS Vaccine Design. Int J Vaccines Vaccin 1(3): 00014. DOI: 10.15406/ijvv.2015.01.00014.

Paradiso, P. R., Rhode, S. L., and Singer, S. L. (1982). Canine parvovirus: A biochemical and ultrastructural characterization. J. Gen. Virol. 62, 113115.

Parrish, C. R. 1990. Emergence, natural history, and variation of canine, mink, and feline parvoviruses. Adv. Virus Res. 38:403-450. 
Pettersen EF, Goddard TD, Huang CC, Couch GS, Greenblatt DM, Meng EC, Ferrin TE (2004). UCSF Chimera--a visualization system for exploratory research and analysis. J Comput Chem. 25(13):1605-12

Singh H, Ansari HR, Raghava GPS (2013) Improved Method for Linear B-Cell Epitope Prediction Using Antigen's Primary Sequence. PLoS ONE 8(5): e62216.

Strassheim, M. L., Gruenberg, A., Veijalainen, P., Sgro, J. Y., and Parrish,
C. R. (1994). Two dominant neutralizing antigenic determinants of Canine Parvovirus are found on the threefold spike of the virus capsid. Virology 198, 175-184.

Tsao J., Chapman M.S., Agbandje M., Keller W., Smith K., Wu H., Luo M., Smith T.J., Rossmann M.G., Compans R.W., et al., The three-dimensional structure of canine parvovirus and its functional implications. Science. 1991; 251: 14561464. doi: $10.1126 /$ science. 2006420 .

\section{How to cite this article:}

Shalini Sharma, Praseenjit Dhar, Inderjeet Singh Yadav and Mandeep Sharma. 2020. B-Cell Linear Epitope Prediction on VP2 Partial Coat Protein Predicted Model of CPV-2b Isolate using Bioinformatics. Int.J.Curr.Microbiol.App.Sci. 9(04): 1154-1159.

doi: https://doi.org/10.20546/ijcmas.2020.904.136 